\title{
UNBOUNDED LOEB MEASURES ${ }^{1}$
}

\section{WARD HENSON}

\begin{abstract}
Uniqueness of the Caratheodory extension is proved for the unbounded case of Loeb's measure space construction in nonstandard analysis and these measures are studied. Methods are developed for handling sets in the $\sigma$-algebra generated by the internal sets. A quantitative theory of degrees of null measure is developed.
\end{abstract}

In [5] Loeb described a measure construction is nonstandard analysis which has proved to be of considerable importance. (See [1] [2] and [4], and forthcoming work of Keisler on stochastic differential equations.) This construction starts with an internal algebra of sets $\mathcal{Q}$ (based on an internal set $X$ ) and an internal, finitely additive measure $\mu: \mathbb{Q} \rightarrow{ }^{*} \mathbf{R}^{+}$, both in some $\boldsymbol{\aleph}_{1}$-saturated nonstandard extension $* \mathfrak{K}$. An ordinary measure ${ }^{o} \mu$ is defined on $\mathbb{Q}$ by:

$$
{ }^{o} \mu(A)= \begin{cases}\operatorname{st}(\mu(A)) & \text { if } \mu(A) \text { is finite in }{ }^{*} \mathbf{R} \\ \infty & \text { otherwise. }\end{cases}
$$

(Here st denotes the standard part map on the finite part of ${ }^{*} \mathbf{R}$.) Then ${ }^{o} \mu$ can be extended by the Carathéodory procedure to a countably additive measure on the $\sigma$-algebra $\sigma(\mathscr{Q})$ generated on $X$ by the sets in $\mathbb{Q}$. Loeb also showed that when ${ }^{\circ} \mu$ is a finite measure this extension to $\sigma(\mathbb{Q})$ is unique. Here we prove that this uniqueness also holds in the unbounded case. This answers a question raised by Loeb (see [5, Footnote 2]).

Unbounded Loeb measures arise naturally even when one starts with a finite measure. For example, if $\mathbb{Q}$ and $\mu$ are given with $\mu(X)$ finite, it is useful to consider the measures ${ }^{\circ}(1 / p \cdot \mu)$ where $p$ is infinitesimal. We will use these measures below to develop a quantitative theory of degrees of null measure for the measure ${ }^{\circ} \mu$.

In this paper and also in [3] a central underlying theme concerns methods for handling sets which are in the $\sigma$-algebras generated by internal sets. Such sets arise not only in connection with Loeb measures but also in many other settings. They are the simplest external sets and any general methods for dealing with them are likely to be widely useful. The techniques used here depend on the simple observation that any set in $\sigma(\mathscr{Q})$ is constructed from a

Received by the editors January 27, 1978 and, in revised form, May 26, 1978.

AMS (MOS) subject classifications (1970). Primary 02H25, 28A05, 28A10.

${ }^{1}$ This research was supported by a grant from the National Science Foundation. 
countable number of sets in $\mathcal{Q}$. In [3] we use the Souslin operation to give an explicit representation of the sets in $\sigma(\mathbb{Q})$.

We adopt here the usual framework of nonstandard analysis (see [9]) and we assume familiarity with Loeb's basic paper [5]. Throughout this paper we use the following notation: $\Re$ is a standard set theoretic superstructure containing the real field $\mathbf{R}$ and $* \Re$ is an $\aleph_{1}$-saturated nonstandard extension of $\Re$.

From now on we consider a fixed internal set $X$ in $* \mathfrak{T}$, a fixed internal algebra $\mathcal{Q}$ of subsets of $X$ (which contains $X$ ) and a fixed internal, finitely additive measure $\mu$ : $\mathbb{Q} \rightarrow{ }^{*} \mathbf{R}^{+}$. (Automatically $\mathbb{Q}$ is closed under internal ${ }^{*}$-finite unions and $\mu$ is *-finitely additive.) Given any family of sets $\mathbb{Q}_{0} \subseteq Q, \sigma\left(Q_{0}\right)$ denotes the $\sigma$-algebra of subsets of $X$ which is generated by $\Theta_{0}$.

Now suppose $S$ is a set in the $\sigma$-algebra $\sigma(Q)$. It is easy to show that there exists a countable family $\mathbb{Q}_{0} \subseteq \mathbb{Q}$ such that $S \in \sigma\left(Q_{0}\right)$. It is no loss of generality to assume that $\mathscr{Q}_{0}$ is a subalgebra of $Q$ (i.e. $\mathcal{Q}_{0}$ is closed under finite unions, finite intersections and complements relative to $X$.) This simple observation plus the saturation condition on $* \mathfrak{T}$ are the keys to what is proved in this paper.

It turns out to be as easy to handle a larger $\sigma$-algebra of sets than $\sigma(\mathbb{Q})$, including sets which are each determined by a countable number of sets from $Q$ in a more general sense. Recall that an algebra $\mathscr{B}$ of subsets of $X$ is complete if it is not only closed under complements (relative to $X$ ), finite unions and finite intersections, but is also closed under unions or intersections of arbitrary collections.

Definition. A set $S \subseteq X$ is countably determined over $\mathbb{Q}$ if there exists a countable subalgebra $\mathbb{Q}_{0}$ of $\mathscr{Q}$ such that $S$ is in the complete algebra of subsets of $X$ which is generated by $\mathbb{Q}_{0}$.

Denote by $\mathcal{C}(\mathbb{Q})$ the collection of all sets $S \subseteq X$ which are countably determined over $\mathbb{Q}$. An easy argument shows that $\mathcal{C}(\mathcal{Q})$ is a $\sigma$-algebra and $\mathcal{C}(\mathbb{Q}) \supseteq \sigma(\mathbb{Q})$. The main technical fact about $\mathcal{C}(\mathbb{Q})$, through which we apply the $\aleph_{1}$-saturation principle, is the following:

Lemma 1. Let $\mathscr{C}_{0}$ be a countable subalgebra of $\mathbb{Q}$ and let $\mathfrak{B}_{0}$ be the complete algebra of subsets of $X$ which is generated by $\mathcal{Q}_{0}$. If $S \subseteq X$ and for each $A \in \mathbb{Q}_{0}$ either $S \subseteq A$ or $S \cap A=\varnothing$, then for each $B \in \mathscr{B}_{0}$, either $S \subseteq B$ or $S \cap B$ $=\varnothing$.

Proof. Let $\mathscr{P}$ be the collection of all nonempty sets $P \subseteq X$ which can be represented in the form $P=\cap_{n} B_{n}$, where $B_{1}, B_{2}, \ldots$ are in $Q_{0}$ and each set in $\mathbb{Q}_{0}$ or its complement occurs in the list. If $P \in \mathscr{P}$ and $A \in \mathbb{Q}_{0}$, then either $P \subseteq A$ or $P \cap A=\varnothing$. Hence $A=\cup\{P \in \mathscr{P} \mid P \subseteq A\}$ whenever $A \in \mathbb{Q}_{0}$. Moreover, $\mathscr{P}$ is a partition of $X$. It follows that $\mathscr{B}_{0}$ consists exactly of those sets $B \subseteq X$ which can be written as $B=\cup^{\mathcal{P}^{\prime}}$ for some $\mathscr{P}^{\prime} \subseteq \mathscr{P}$. Thus if 
$B \in \mathscr{B}_{0}$ and $P \in \mathcal{P}$, then either $P \subseteq B$ or $P \cap B=\varnothing$.

Now suppose $S \subseteq X$ and for each $A \in \mathbb{Q}_{0}$, either $S \subseteq A$ or $S \cap A=\varnothing$. Let $B_{1}, B_{2}, \ldots$ be a list of all elements of $Q_{0}$ which contain $S$. Since each element of $Q_{0}$ or its complement is in this list, $P=\cap_{n} B_{n}$ is in $\mathcal{P}$. But $S \subseteq P$ and therefore, if $B \in \mathscr{B}_{0}$ we conclude that either $S \subseteq B$ (if $P \subseteq B$ ) or $S \cap B=\varnothing$ (if $P \cap B=\varnothing$ ).

We remark here that the use of countable subalgebras of $\mathscr{Q}$ in the definition of $\mathcal{C}(Q)$ is dictated by the assumption that $* \Re$ is only $\aleph_{1}$-saturated. More generally, if $* \Re$ is $\kappa$-saturated, then we can take $\mathcal{C}(\mathbb{Q})$ to be the family of all $S \subseteq X$ such that $S$ is in the complete algebra generated by some subalgebra of $\mathcal{Q}$ which has cardinality $<\kappa$. In this setting, all the results below remain true, with the same proofs as given here.

THEOREM 1. For each set $S$ in $\mathcal{C}(\mathbb{Q})$, exactly one of the following conditions holds (in particular this is true whenever $S$ is in $\sigma(\mathbb{Q})$ ).

(i) There exists $A \in \mathbb{Q}$ such that $A \subseteq S$ and $\mu(A)$ is infinite.

(ii) There exist $\left(A_{n} \mid n \in \mathbf{N}\right)$ in $Q$ such that $S \subseteq \cup_{n} A_{n}$ and $\mu\left(A_{n}\right)$ is finite for each $n \in \mathbf{N}$.

Proof. Let $S \in \mathcal{C}(\mathbb{Q})$ and let $\mathbb{Q}_{0}$ be a countable subalgebra of $\mathbb{Q}$ which determines $S$. Let $Q_{0}^{\prime}=\left\{A \in \mathbb{Q}_{0} \mid \mu(A)\right.$ is finite $\}$. If $\mathbb{Q}_{0}^{\prime}$ covers $S$ then condition (ii) holds. If not, there exists $p \in S$ such that $p \notin \cup \mathbb{Q}_{0}^{\prime}$. Let $Q_{0}^{\prime \prime}=\left\{B \in \mathbb{Q}_{0} \mid p \in B\right\}$; then $\mathbb{Q}_{0}^{\prime \prime}$ is closed under finite intersections and $\mu(B)$ is infinite for each $B \in \mathbb{Q}_{0}^{\prime \prime}$. Thus the countable family of all sets

$$
S(n, B)=\{A \in \mathbb{Q} \mid p \in A \subseteq B \text { and } \mu(A) \geqslant n\}
$$

(where $n \in \mathbf{N}$ and $B \in \mathcal{Q}_{0}^{\prime \prime}$ ) has the finite intersection property. Since $* \mathfrak{N}$ is $\aleph_{1}$-saturated there exists $A \in \mathbb{Q}$ such that $A \in S(n, B)$ for all $n \in \mathbf{N}$ and $B \in \mathbb{Q}_{0}^{\prime \prime}$. That is, $\mu(A)$ is infinite, $p \in A$ and $A \subseteq B$ for all $B \in \mathbb{Q}_{0}^{\prime \prime}$. Consider an arbitrary set $B$ from $Q_{0}$. If $A \_B$, it follows that $p \notin B$, so $p \in X \backslash B$. That is, $X \backslash B \in \mathbb{Q}_{0}^{\prime \prime}$ and hence $A \subseteq X \backslash B$. Therefore, for any $B \in \mathbb{Q}_{0}$, either $A \subseteq B$ or $A \cap B=\varnothing$. Since $S$ is determined by $\mathbb{Q}_{0}$ it follows that $A \subseteq S$ or $A \cap S=\varnothing$. Since $p \in A \cap S$, it must be that $A \subseteq S$, showing that condition (i) holds.

Finally we must show that (i) and (ii) cannot both hold. If they do, then $A \subseteq \cup_{n} A_{n}$; but since these sets are internal and $* \Re$ is $\aleph_{1}$-saturated, we must have $A \subseteq A_{1} \cup \cdots \cup A_{k}$ for some $k \in \mathbf{N}$. This is impossible, since $\mu(A)$ is infinite while each $\mu\left(A_{n}\right)$ is finite.

COROllary 1. The extension of ${ }^{\circ} \mu$ to a countably additive measure on $\sigma(\mathbb{Q})$ is unique.

Proof. Suppose $\nu_{1}$ and $\nu_{2}$ are countably additive measures on $\sigma(\mathscr{Q})$ which agree with ${ }^{\circ} \mu$ on $Q$. Let $S \in \sigma(\mathbb{Q})$; since $\sigma(\mathbb{Q}) \subseteq \mathcal{C}(\mathbb{Q})$ we may apply Theorem 1. If condition (i) holds, there exists $A \in \mathbb{Q}$ such that $A \subseteq S$ and ${ }^{\circ} \mu(A)=\infty$. Therefore $\nu_{j}(S)=\infty$ for $j=1$ and $j=2$. Otherwise condition 
(ii) holds and there exists $\left(A_{n} \mid n \in \mathrm{N}\right)$ in $Q$ such that each $\mu\left(A_{n}\right)$ is finite in ${ }^{*} \mathbf{R}$ and $S \subseteq \cup_{n} A_{n}$. We may suppose $A_{1} \subseteq A_{2} \subseteq \ldots$, taking finite unions if necessary. Now we may apply [5, Theorem 1], as discussed above, obtaining that for $j=1,2$

$$
\nu_{j}\left(S \cap A_{n}\right)=\inf \left\{{ }^{\circ} \mu(A) \mid A \in \mathbb{Q} \text { and } S \cap A_{n} \subseteq A \subseteq A_{n}\right\} .
$$

In particular, $\nu_{1}\left(S \cap A_{n}\right)=\nu_{2}\left(S \cap A_{n}\right)$ for all $n \in \mathbf{N}$. Since $S \cap A_{1} \subseteq S \cap$ $A_{2} \subseteq \ldots$ and $S=\cup_{n}\left(S \cap A_{n}\right)$, it follows that $\nu_{1}(S)=\nu_{2}(S)$. That is, $\nu_{1}=\nu_{2}$ on all of $\sigma(Q)$.

It is convenient to let ${ }^{o} \mu$ also denote the unique extension of ${ }^{o} \mu$ from $\mathcal{Q}$ to all of $\sigma(Q)$. The next result gives another useful way of viewing this extension measure.

COROllary 2. For each set $S$ in $\sigma(Q)$, exactly one of the following conditions holds:

(i) There exists $A \in \mathbb{Q}$ such that $S \subseteq A$ and ${ }^{\circ} \mu(A)$ is finite (and thus ${ }^{\circ} \mu(S)$ is finite).

(ii) For each $n \in \mathbf{N}$ there exists $A_{n} \in \mathbb{Q}$ such that $A_{n} \subseteq S$ and ${ }^{\circ} \mu\left(A_{n}\right) \geqslant n$ (and thus ${ }^{\circ} \mu(S)=\infty$ ).

Proof. Fix $S \in \sigma(\mathbb{Q})$. If $S$ has a subset $A \in \mathbb{Q}$ which has infinite measure, then obviously condition (ii) holds. Otherwise, by Theorem 1 , there are sets $\left(A_{n} \mid n \in \mathbf{N}\right)$ in $\mathbb{Q}$ such that $S \subseteq \cup_{n} A_{n}$ and each $\mu\left(A_{n}\right)$ is finite in ${ }^{*} \mathbf{R}$. Applying [5, Theorem 1] to the restriction of $(Q, \mu)$ to $A_{n}$, we have that there exist sets $B_{n}, C_{n}$ in $\mathscr{Q}$ such that $B_{n} \subseteq S \cap A_{n} \subseteq C_{n} \subseteq A_{n}$ and $\mu\left(C_{n} \backslash B_{n}\right) \leqslant$ $2^{-n}$, for each $n \in \mathbf{N}$.

If the numbers $\mu\left(B_{1} \cup \cdots \cup B_{n}\right)$, for $n \in \mathbf{N}$, are not uniformly bounded above by an element of $\mathbf{N}$, then condition (ii) evidently holds. Otherwise there exists a fixed $k \in \mathbf{N}$ so that for all $n \in \mathbf{N}$

$$
\mu\left(B_{1} \cup \cdots \cup B_{n}\right) \leqslant k \text {. }
$$

Hence

$$
\mu\left(C_{1} \cup \cdots \cup C_{n}\right) \leqslant k+2^{-1}+2^{-2}+\cdots+2^{-n} \leqslant k+1
$$

for all $n \in \mathbf{N}$. Since ${ }^{*} \mathfrak{R}$ is $\boldsymbol{\aleph}_{1}$-saturated, there must exist a set $C \in \mathbb{Q}$ such that $\mu(C) \leqslant k+1$ and $C_{n} \subseteq C$ for all $n \in \mathbf{N}$. Therefore $\mu(C)$ is finite and

$$
S=\bigcup_{n}\left(S \cap A_{n}\right) \subseteq \bigcup_{n} C_{n} \subseteq C .
$$

Thus condition (i) holds, and the proof is complete.

We now turn to a closer analysis of the sets of ${ }^{\circ} \mu$ measure 0 , made possible by Theorem 1 and its corollaries. As a measure of size we take a set $\Delta$ of infinitesimals $\delta \geqslant 0$ satisfying

(a) $0 \leqslant \eta<\delta \in \Delta$ implies $\eta \in \Delta$,

(b) $\delta, \eta \in \Delta$ implies $\delta+\eta \in \Delta$. 
Note that (a), (b) imply that if $p \in{ }^{*} \mathbf{R}$ and $p>\Delta$, then also $p / n>\Delta$ for every $n \in \mathbf{N}$. (Here write $p>\Delta$ to mean $p>q$ for every $q \in \Delta$.)

Definition. A set $S \subseteq X$ is a $\Delta$-null set relative to $(Q, \mu)$ if for each $p \in{ }^{*} \mathbf{R}, p>\Delta$ implies that there exists $A \in \mathbb{Q}$ such that $S \subseteq A$ and $\mu(A)<$ $p$.

Another way of putting the definition is this: $S$ is a $\Delta$-null set if it is a null set for the measure ${ }^{\circ}(1 / p \cdot \mu)$ for every $p>\Delta$. This makes it clear that the family of all $\Delta$-null sets is a $\sigma$-ideal of subsets of $X$ (i.e. it is closed under taking subsets and countable unions). We denote this $\sigma$-ideal of $\Delta$-null sets by $\Re_{\Delta}$.

We will call $\Delta$ Archimedean if there exists $p \in{ }^{*} \mathbf{R}^{+}$such that

$$
\Delta=\{q \geqslant 0 \mid q / p \text { is infinitesimal }\} .
$$

That is, this requires that $\Delta$ be just the set of positive infinitesimals in ${ }^{*} \mathbf{R}$ multiplied by $p$, and so $p, p / 2, p / 3, \ldots$ is a decreasing sequence of numbers which is cofinal (downward) among the numbers greater than $\Delta$. In this case, $\mathscr{H}_{\Delta}$ is simply the $\sigma$-ideal of null sets for the measure ${ }^{\circ}(1 / p \cdot \mu)$ and nothing essentially new is involved.

If $\Delta$ is non-Archimedean then for each $p>\Delta$ there must exist $q>\Delta$ such that $p / q$ is infinite, since $p, p / 2, p / 3, \ldots$ is not cofinal above $\Delta$ as in the Archimedean case. In some contexts a non-Archimedean interval arises in a completely natural way and is very useful for technical purposes. For example, in Robinson's development of asymptotic analysis, a basic role is played by intervals of the form

$$
\Delta=\left\{p \geqslant 0 \mid p<\delta^{n} \text { for all } n \in \mathbf{N}\right\}
$$

where $\delta>0$ is a fixed infinitesimal. (See [8], [6] and [7].) All such intervals are non-Archimedean.

THEOREM 2. For each $S$ in $\sigma(\mathbb{Q}), S$ is in $\mathfrak{B}_{\Delta}$ if and only if for each $A \in \mathbb{Q}$, $A \subseteq S$ implies $\mu(A) \in \Delta$. If $\Delta$ is non-Archimedean, then this equivalence holds for all $S \in \mathcal{C}(\mathbb{Q})$.

Proof. If $\Delta$ is Archimedean, then $\mathscr{乃}_{\Delta}$ is the $\sigma$-ideal of $o(1 / p \cdot \mu)$-null sets for some $p$. In this case Theorem 2 is just a restatement of Loeb's result [5, Theorem 1].

For the rest of the proof we assume that $\Delta$ is non-Archimedean and that $S$ is in $\mathcal{C}(\mathbb{Q})$.

Suppose $S$ is in $\mathscr{H}_{\Delta}, A \in \mathbb{Q}$ and $A \subseteq S$. Evidently $\mu(A)<p$ must hold for every $p>\Delta$, since there exists $B \in \mathbb{Q}$ with $S \subseteq B$ and $\mu(B)<p$. Since $\mu(A)$ is in ${ }^{*} \mathbf{R}^{+}$, it must therefore be in $\Delta$.

Conversely, suppose $S \in \mathcal{C}(\mathbb{Q})$ and also $\mu(A) \in \Delta$ whenever $A \in \mathbb{Q}$ and $A \subseteq S$. Given $p>\Delta$, we may choose $q>\Delta$ so that $p / q$ is infinite. We apply Theorem 1 to $S$ and the internal measure space $(Q, 1 / q \cdot \mu)$. This shows that 
there must exist $A_{1} \subseteq A_{2} \subseteq \ldots$ in $Q$ such that $S \subseteq \cup_{n} A_{n}$ and each $1 / q$. $\mu\left(A_{n}\right)$ is finite. Since $* \mathscr{N}$ is $\aleph_{1}$-saturated, there exists $A \in \mathbb{Q}$ satisfying $A_{n} \subseteq A$ for all $n \in \mathbf{N}$ and $1 / q \cdot \mu(A)<p / q$. That is, $\mu(A)<p$ and $S \subseteq$ $\cup_{n} A_{n} \subseteq A$. Since $p>\Delta$ was arbitrary, this shows that $S$ is in $\mathscr{B}_{\Delta}$.

In many cases $\mathscr{B}_{\Delta}$ has a strong closure property which might be loosely termed closure under "*-finite unions". This was noticed by H. J. Keisler after we told him about the results above and he has generously agreed to let us include it here.

Let $F$ be a ${ }^{*}$-finite set in ${ }^{*} \Re$ of (internal) cardinality $\tau \in{ }^{*} \mathbf{N}$. We think of a subset $Y$ of $F \times X$ as being (essentially) an enumeration $\left(Y_{a} \mid a \in F\right)$ of subsets of $X$, where

$$
Y_{a}=\{x \in X \mid\langle a, x\rangle \in Y\}
$$

for each $a \in F$.

Now let $\mathscr{Q}$ be the internal algebra of subsets of $F \times X$ generated in $* \Re$ by sets of the form $E \times A$, where $E \subseteq F, E$ is internal and $A \in \mathbb{Q}$. Equivalently, $\mathbb{Q}$ is the algebra of all internal sets $H \subseteq F \times X$ such that $H_{a} \in \mathbb{Q}$ for every $a \in F$.

THEOREM 3 (KeISLER). Assume $\Delta$ is closed under multiplication by $\tau=|F|$. If $Y \subseteq F \times X, Y \in \mathcal{C}\left(\mathbb{Q}^{\prime}\right)$ and if $Y_{a} \in \mathscr{B}_{\Delta}$ for each $a \in F$, then $\cup\left\{Y_{a} \mid a \in F\right\}$ is also in $\mathfrak{B}_{\Delta}$.

Proof. We define an internal finitely additive measure $\mu^{\prime}$ on $\mathbb{Q}^{\prime}$ by

$$
\mu^{\prime}(H)=\sum_{a \in F} \mu\left(H_{a}\right) .
$$

Note that $\Delta$ must be non-Archimedean since it is closed under multiplication by $\tau$. Thus we can apply Theorem 2 to $\left(\mathbb{Q}^{\prime}, \mu^{\prime}\right)$ and $\Delta$.

Let $H \in \mathbb{Q}^{\prime}$ be such that $H \subseteq Y$. Thus $H_{a} \subseteq Y_{a} \in \mathscr{B}_{\Delta}$ for each $a \in F$. It follows that $\mu\left(H_{a}\right) \in \Delta$ holds for each $a \in F$. Since the function taking $a$ to $\mu\left(H_{a}\right)$ is internal, we may set

$$
\delta=\max \left\{\mu\left(H_{a}\right) \mid a \in F\right\}
$$

and we have $\delta \in \Delta$. Then

$$
\mu^{\prime}(H)=\sum_{a \in F} \mu\left(H_{a}\right) \leqslant \delta \tau \in \Delta .
$$

Applying Theorem 2 we conclude that $Y$ is a $\Delta$-null set relative to $\left(\mathbb{Q}^{\prime}, \mu^{\prime}\right)$.

Therefore, given $p>\Delta$ there exists $H \in \mathbb{Q}^{\prime}$ so that $Y \subseteq H$ and $\mu^{\prime}(H)<p$. Then

$$
\bigcup\left\{Y_{a} \mid a \in F\right\} \subseteq \bigcup\left\{H_{a} \mid a \in F\right\} \in \mathbb{Q}
$$

since $\mathscr{Q}$ is closed under internal, ${ }^{*}$ finite unions. Also

$$
\mu\left(\bigcup\left\{H_{a} \mid a \in F\right\}\right) \leqslant \sum_{a \in F} \mu\left(H_{a}\right)=\mu^{\prime}(H)<p
$$


since $\mu$ is *-finitely additive. This shows that $U\left\{Y_{a} \mid a \in F\right\}$ is in $\mathscr{B}_{\Delta}$, completing the proof.

As an example of an interval $\Delta$ to which Theorem 3 applies, consider the interval

$$
\Delta=\left\{p \geqslant 0 \mid p<\delta^{n} \text { for all } n \in \mathbf{N}\right\}
$$

introduced by Robinson [8], [6]. This $\Delta$ is closed under multiplication by $\tau=|F|$ as long as $\tau<\delta^{-n}$ holds for some $n \in \mathbf{N}$.

We next give a result concerning the ${ }^{\circ} \mu$-measurability of the sets $\cup\left\{Y_{a} \mid a\right.$ $\in F\}$ when ${ }^{\circ} \mu$ is a finite measure. Loosely speaking, it states that the $\sigma$-algebra of ${ }^{\circ} \mu$-measurable sets is closed under "*-finite unions."

TheOREM 4. Assume that $\mu(X)$ is finite. If $Y \subseteq F \times X$ and $Y \in \sigma\left(\mathbb{Q}^{\prime}\right)$, then $\cup\left\{Y_{a} \mid a \in F\right\}$ is ${ }^{\circ} \mu$-measurable.

Proof. Since ${ }^{\circ} \mu$ is a finite measure, the collection of ${ }^{\circ} \mu$-measurable sets is closed under the Souslin operation. Thus it suffices to show that $\cup\left\{Y_{a} \mid a \in\right.$ $F$ \} can be derived from sets in $Q$ by the Souslin operation. The proof of this is much like the proof of Theorem 1 in [3]. Here, instead of a standard part map, we make use of the coordinate projection $\pi: F \times X \rightarrow X$ defined by $\pi(a, x)=x$. First observe that if $H$ is an internal set in $Q^{\prime}$ then $\pi(H)$, which equals $\cup\left\{H_{a} \mid a \in F\right\}$, is an element of $\mathcal{Q}$. Also, if $H_{1} \supseteq H_{2} \supseteq \ldots$ are in $\mathcal{Q}^{\prime}$, then

$$
\pi\left(\bigcap_{n} H_{n}\right)=\bigcap_{n} \pi\left(H_{n}\right)
$$

(The right side contains $x$ only if $A \times\{x\}$ intersects each $H_{n}$; since $* \mathfrak{N}$ is $\aleph_{1}$-saturated, this is the same as saying that $A \times\{x\}$ intersects $\bigcap_{n} H_{n}$.)

Given $Y \in \sigma\left(\mathbb{Q}^{\prime}\right)$, we may represent $Y$ in terms of elements of $\mathbb{Q}^{\prime}$ using the Souslin operation. That is, there exist sets $\left(H_{s} \mid s \in \mathrm{Seq}\right)$ from $\mathbb{Q}^{\prime}$ such that

$$
Y=\bigcup_{\alpha} \bigcap_{n} H_{\alpha \mid n}
$$

(Here Seq is the set of finite sequences from $\mathbf{N}, \alpha$ ranges over all functions from $\mathbf{N}$ to $\mathbf{N}$ and $\alpha \mid n$ is $\alpha(1), \alpha(2), \ldots, \alpha(n)$.) Since $\mathcal{Q}^{\prime}$ is closed under finite intersections we may assume that $H_{s} \supseteq H_{t}$ whenever $s$ is an initial segment of $t$. Then

$$
\bigcup\left\{Y_{a} \mid a \in F\right\}=\pi(Y)=\bigcup_{\alpha} \bigcap_{n} \pi\left(H_{\alpha \mid n}\right)
$$

which is derived from sets in $Q$ by the Souslin operation.

We note that the introduction of the Souslin operation here seems necessary. It can be shown, using an argument like the one given for Theorem 4 in [3], that every set derived by the Souslin operation from sets in $\mathbb{Q}$ can be represented in the form $\pi(Y)$ with $Y \in \sigma\left(Q^{\prime}\right)$.

Also, the proof of Theorem 4 is valid without any change under the weaker assumption that $Y$ can be derived from sets in $\mathbb{Q}^{\prime}$ using the Souslin operation. 


\section{REFERENCES}

1. R. M. Anderson, A non-standard representation for Brownian motion and Itô integration, Israel J. Math. 25 (1976), 15-46.

2. Star-finite probability theory, $\mathrm{Ph}$. D. Thesis, Yale University, 1977.

3. C. Ward Henson, Analytic sets, Baire sets and the standard part map, Canad. J. Math. (to appear).

4. H. J. Keisler, Hyperfinite model theory, Logic Colloquium 76, North-Holland, Amsterdam, 1977, pp. 5-110.

5. P. Loeb, Conversion from nonstandard to standard measure spaces and applications in probability theory, Trans. Amer. Math. Soc. 211 (1975), 113-122.

6. A. H. Lightstone and A. Robinson, Nonarchimedean fields and asymptotic expansions, North-Holland, Amsterdam, 1975.

7. W. A. J. Luxemburg, On a class of valuation fields introduced by A. Robinson, Israel J. Math. 25 (1976), 189-201.

8. A. Robinson, Function theory on some nonarchimedean fields, Papers on the Foundations of Mathematics, Slaught Memorial Papers No. 13, Math. Assoc. Amer., Washington, D. C., 1973, pp. 87-109.

9. K. Stroyan and W. A. J. Luxemburg, Introduction to the theory of infinitesimals, Academic Press, New York, 1976.

Department of Mathematics, University of Illinois at Urbana-Champaign, Urbana, ILLINOIS 61801 